C-A/AP/\#202

May 2005

\title{
Beam-Based Non-Linear Optics Corrections In Colliders
}

F. Pilat, Y. Luo, N. Malitsky, V. Ptitsyn

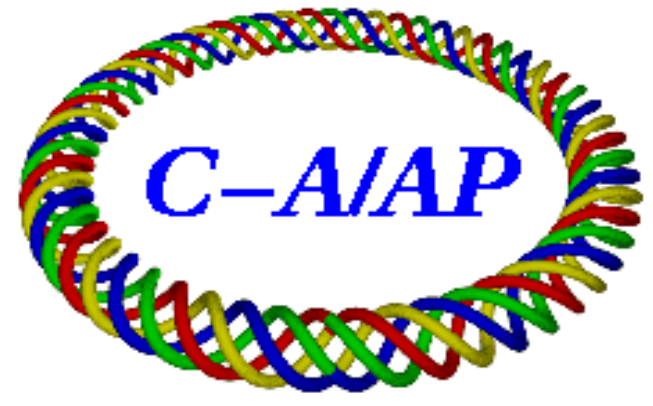

Collider-Accelerator Department Brookhaven National Laboratory

Upton, NY 11973 


\title{
Beam-based non-linear optics corrections in colliders
}

\author{
F. Pilat, Y. Luo, N. Malitsky and V. Ptitsyn \\ Brookhaven National Laboratory
}

\begin{abstract}
A method has been developed to measure and correct operationally the non-linear effects of the final focusing magnets in a collider, that gives access to the multi-pole errors effects by applying closed orbit bumps, and by analyzing the resulting tune and orbit shifts. This technique has been tested and used during 4 years of operations at RHIC, the Relativistic Heavy Ion Collider at BNL. We will discuss here the theoretical basis of the method, the experimental set-up, the correction results, the present understanding of the machine model, and the potential and limitations of the method itself.
\end{abstract}

\section{Introduction}

The IR bump technique has been developed to correct operationally local errors in the interaction regions (IR). The dynamic aperture of a hadron collider is typically determined by beam-beam effects and by the errors from the magnets in the low- $\beta^{*}$ sections. We correct locally IR coupling effects in all IR's, but non-linear effects becomes significant at RHIC only for $\beta^{*}<2 \mathrm{~m}$. The local correction of non-linear effects has the potential of improving machine dynamic aperture, beam lifetime and operability. Non-linear corrections, together with emittance reduction that will be made available by electron cooling, a planned machine upgrade, can open the possibility of RHIC operation from the present design of $\beta^{*} \sim 1 \mathrm{~m}$ to $\beta^{*} \sim 0.5 \mathrm{~m}$, in resulting a $50 \%$ increase in luminosity. We describe here the foundation of the IR bump method with a general analysis valid for all orders, followed by the particular example of application to the sextupole, normal and skew, and octupole effects. In the following chapter we will describe the experimental set-up, procedures and results of the correction achieved so far in RHIC. Sextupole and 
octupole corrections will be described in more detail, followed by a discussion on the feasibility of measurements and correction of higher orders. Finally, we will discuss the present understanding of the sources of non-linear optics distortion, by comparing the beam measurements and correction with magnetic measurements of the IR magnets.

\section{IR Bump method - General discussion}

The magnetic errors in an accelerator magnet can be described in terms of the multipole errors $a_{n}$ and $b_{n}$ defined as:

$$
B_{y}+i B_{x}=B_{N} \sum_{n=0}^{\infty}\left(b_{n}+i a_{n}\right)\left(\frac{x+i y}{R_{r}}\right)^{n}
$$

$B_{N}$ represents the main field of a magnet measured at a reference radius $R_{r}$. For a quadrupole, for instance, $B_{n} / R_{r}$ is the gradient. $b_{n}$ and $a_{n}$ are respectively the normal and skew harmonics of magnet errors.

A local orbit excursion through a region with nonlinear fields generates feed-down effects to lower order field harmonics. The most useful observables come from the feeddown to the zero and first order harmonics, which affect respectively the beam closed orbit and betatron tunes. The magnitude of the orbit and tune changes depends on the size of the orbit excursion. For an orbit bump built with 3 dipole correctors (3-bump), the bump amplitude $A_{\text {bump }}$ is the measure of the orbit excursion and is defined throughout this paper as the orbit excursion at the location of the dipole corrector in the middle of the bump.

The tune shifts measured arise either from the feed-down to the normal gradient or from the repelling effect of linear coupling. The tune shift $\Delta Q$ and the linear coupling term $\Delta c$ for different bump planes ( $\mathrm{H}$ and $\mathrm{V}$ ) and for different multipole errors (normal, skew, even and odd orders) can be expressed as follows:

$$
\begin{array}{ll}
\Delta Q(H, \text { norm })=g\left(b_{n}, x_{c o}\right) ; & \Delta Q(V, \text { norm }, \text { odd })=-1^{(n-1) / 2} g\left(b_{n}, y_{c o}\right) \\
\Delta Q(V, \text { skew }, \text { even })=-1^{n / 2} g\left(a_{n}, y_{c o}\right) ; & \Delta c(H, \text { skew })=h\left(a_{n}, x_{c o}\right) \\
\Delta c(V, \text { norm, even })=-1^{(n-1) / 2} h\left(b_{n}, y_{c o}\right) ; & \Delta c(V, \text { skew }, \text { odd })=-1^{(n+1) / 2} h\left(a_{n}, y_{c o}\right)
\end{array}
$$


where the functions $g$ and $h$ are:

$$
\begin{aligned}
& g\left(c_{n}, z_{c o}\right)=\frac{n}{4 \pi} \frac{1}{B \rho} \int \beta_{z} B_{N} c_{n} \frac{z_{c o}^{n-1}}{R^{n}} d s \\
& h\left(c_{n}, z_{c o}\right)=\frac{n}{2 \pi} \frac{1}{B \rho} \int \sqrt{\beta_{x} \beta_{y}} B_{N} c_{n} \frac{z_{c o}^{n-1}}{R^{n}} e^{i\left(\mu_{x}-\mu_{y}\right)} d s
\end{aligned}
$$

$c_{n}$ stands for either the $a_{n}$ or $b_{n}$ and $z$ stands for $x$ or $y$. The tune shifts for horizontal and vertical tunes are expressed then as: $\Delta Q_{x}=\Delta Q, \Delta Q_{y}=-\Delta Q$.

Whether the feed-down from a given multi-pole affects the normal gradient or the linear coupling depends on the plane of the bump, on whether the multi-pole is normal or skew and on the parity of the multi-pole order $n$ as summarized in Table 1.

Table 1: Measurable quantities for each non-linear multipole

\begin{tabular}{|c|c|c|c|c|c|c|c|}
\hline Bump & $b_{2}$ & $a_{2}$ & $b_{3}$ & $a_{3}$ & $b_{4}$ & $a_{4}$ & $b_{5}$ \\
\hline $\mathrm{H}$ & $\Delta Q$ & $\Delta c$ & $\Delta Q$ & $\Delta c$ & $\Delta Q$ & $\Delta c$ & $\Delta Q$ \\
\hline $\mathrm{V}$ & $\Delta c$ & $\Delta Q$ & $\Delta Q$ & $\Delta c$ & $\Delta c$ & $\Delta Q$ & $\Delta Q$ \\
\hline
\end{tabular}

In order to simplify the identification of individual multipoles from the observed tune shifts, the measurements conditions should be such that the tune shifts produced by coupling are negligible compared with the tune shifts from the normal gradient change. For this condition to be valid, the residual coupling in the machine has to be well corrected, and the horizontal and vertical tunes have to be well separated. The degree of the coupling compensation and the tune separation must satisfy the relation:

$$
\frac{\left|C_{0}+\Delta c\right|^{2}}{4 \Delta Q_{x y}}<<\Delta Q
$$

Where $C_{0}$ is the residual component of the coupling and $\Delta Q_{x y}=\left|\left\{Q_{x}\right\}-\left\{Q_{y}\right\}\right|$ is the betatron tune separation. 
As seen from Table 1 some of the nonlinear errors, like the $a_{3}$ error, do not produce tune shifts due to the normal gradient feed-down when either horizontal or vertical orbit bump is used. We will show in the next section that in these cases a diagonal bump can be used.

\section{IR bump method - multipole by multipole correction}

Here we apply the IR bump relations, (2) and (3), explicitly to low-order non-linear errors to allow easier discussion of the experimental results for sextupole and octupole discussed later. The essence of these corrections is the minimization, respectively; of the linear and quadratic tune shifts caused by an orbit bump. In the RHIC experimental setup the bump amplitude changes in a continuous way and the polynomial coefficients for the dependence of the tune shifts with bump amplitudes are obtained by polynomial fitting. Here we discuss explicitly the sextupole, skew sextupole, octupole, and skew octupole nonlinear effects and will show that the correction of the tune shift versus bump amplitude leads to the compensation of resonance strength produced by the given nonlinearities. Two assumptions are crucial for this analysis. First, that the betatron phase advances across the triplets on each side of the IR are very small. Thus for a given IR triplet the sources of nonlinear errors as well as corresponding nonlinear correctors can be considered as located at the same betatron phase. The second assumption is that the betatron phase advance over the interaction region between the triplets is very close to $\pi$. Both assumptions are typical for low- $\beta^{*}$ IR's and certainly are satisfied at RHIC.

\section{Sextupole effects: resonances and tune shifts}

The perturbation Hamiltonian for a sextupole error is given by

$$
\Delta H=\frac{k_{2}}{6} \cdot\left(x^{3}-3 x y^{2}\right)
$$

The sextupole strength $k_{2}$ is proportional to the $b_{2}$ magnet error and also describes the sextupole corrector strengths,

$$
k_{2}=\frac{1}{(B \rho)} \frac{\partial^{2} B_{y}}{\partial x^{2}}=\frac{2 B_{N} b_{2}}{(B \rho) R_{r}^{2}}
$$


In order to check the resonance strengths, we use action-angle coordinates,

$$
\left\{\begin{array}{l}
x=\sqrt{2 J_{x} \beta_{x}} \cos \psi_{x} \\
y=\sqrt{2 J_{y} \beta_{y}} \cos \psi_{y}
\end{array}\right.
$$

where $J_{x, y}$ and $\psi_{x, y}$ are the horizontal and vertical action and angles. Substituting (7) into (5) and expanding, shows that normal sextupoles can drive the $Q_{x}=p, 3 Q_{x}=p$, and the $Q_{x} \pm 2 Q_{y}=p$ resonances. For full correction, the following resonance strengths should be minimized simultaneously:

$$
\begin{array}{ll}
\sum_{j}\left[k_{2} \beta_{x}^{1 / 2} \beta_{y} d s \exp \left(i \psi_{x}\right)\right]_{j} \rightarrow 0 ; & \sum_{j}\left[k_{2} \beta_{x}^{3 / 2} d s \exp \left(i \psi_{x}\right)\right]_{j} \rightarrow 0 \\
\sum_{j}\left[k_{2} \beta_{x}^{3 / 2} d s \exp \left(i 3 \psi_{x}\right)\right]_{j} \rightarrow 0 ; \quad & \sum_{j}\left[k_{2} \beta_{x}^{1 / 2} \beta_{y} d s \exp \left(i\left(\psi_{x} \pm 2 \psi_{y}\right)\right)\right]_{j} \rightarrow 0
\end{array}
$$

The sums are taken over all quadrupoles and sextupole correctors in the IR. The correction weight factors for the resonances are $\beta_{x}^{3 / 2}$ and $\beta_{x}^{1 / 2} \beta_{y}$. In the approximation of negligible betatron phase advances in the triplets and $\pi$ phase advances between the triplets, from Eq. (8) one obtains:

$$
\left\{\begin{array}{l}
\sum_{L} k_{2} \beta_{x}^{3 / 2} d s-\sum_{R} k_{2} \beta_{x}^{3 / 2} d s=0 \\
\sum_{L} k_{2} \beta_{x}^{1 / 2} \beta_{y} d s-\sum_{R} k_{2} \beta_{x}^{1 / 2} \beta_{y} d s=0
\end{array}\right.
$$

where $L$ and $R$ stand for the left and the right side triplet. Notice that for a round beam, the $3 Q_{x}=p$ and $Q_{x} \pm 2 Q_{y}=p$ resonances are the leading resonances respectively in the horizontal and vertical planes.

According to Table 1, a horizontal orbit bump should be used to produce the tune shift due to normal gradient feed-down from sextupoles. The tune shifts from a sextupole is proportional to the horizontal beam orbit offsets,

$$
\left\{\begin{array}{l}
\Delta Q_{x}=\frac{1}{4 \pi} \sum k_{2} \beta_{x} x_{c o} d s \\
\Delta Q_{y}=-\frac{1}{4 \pi} \sum k_{2} \beta_{y} x_{c o} d s
\end{array}\right.
$$


Again in the approximation of small phase advances in the triplets and the $\pi$ phase advance between the triplets, the horizontal orbit in the triplet from an IR bump is approximately proportional to $\beta_{x}^{1 / 2}$ :

$$
\begin{aligned}
& x_{c o} \square A_{b u m p} \frac{\beta_{x}^{1 / 2}}{\beta_{x c}^{1 / 2}}, \text { in a triplet on one side of the IR } \\
& x_{c o} \square-A_{b u m p} \frac{\beta_{x}^{1 / 2}}{\beta_{x c}^{1 / 2}} \text {, in a triplet on the other side of the IR }
\end{aligned}
$$

where $A_{b u m p}$ is the orbit bump amplitude and $\beta_{c x}$ is the beta-function at location of the central corrector of the bump. The total tune shift from the sextupoles in the triplets region from one IR, due to the horizontal IR bump are then given by:

$$
\left\{\begin{array}{l}
\Delta Q_{x} \propto\left(\sum_{L} k_{2} \beta_{x}^{3 / 2} d s-\sum_{R} k_{2} \beta_{x}^{3 / 2} d s\right) \cdot \frac{A_{\text {bump }}}{4 \pi \beta_{x c}^{1 / 2}} \\
\Delta Q_{y} \propto-\left(\sum_{L} k_{2} \beta_{x}^{1 / 2} \beta_{y} d s-\sum_{R} k_{2} \beta_{x}^{1 / 2} \beta_{y} d s\right) \cdot \frac{A_{\text {bump }}}{4 \pi \beta_{x c}^{1 / 2}}
\end{array}\right.
$$

The sextupole field errors in the triplets can be corrected by minimizing the linear coefficients of the tune shifts with the horizontal bump amplitude with two local sextupole correctors. Comparing (11) and (9), one concludes that for a normal sextupole the tune shift correction is equivalent to the resonance compensation.

\section{Skew sextupole effects: resonances and tune shifts}

The perturbation Hamiltonian for a skew sextupole error is given by

$$
\Delta H=\frac{k_{2 s}}{6}\left(3 x^{2} y-y^{3}\right)
$$

where the skew sextupole strength $k_{2 s}$ is proportional to the $a_{2}$ magnet error:

$$
k_{2 s}=\frac{1}{(B \rho)} \frac{\partial^{2} B_{x}}{\partial x^{2}}=\frac{2 B_{N} a_{2}}{(B \rho) R_{r}^{2}}
$$


By substituting (7) into (12) and expanding, it follows that skew sextupoles can generate the $Q_{y}=p, 2 Q_{x} \pm Q_{y}=p$, and $3 Q_{y}=p$ resonances. The correction of these resonances requires the minimization of the following resonance strengths:

$$
\begin{array}{ll}
\sum_{j}\left[\beta_{x} \beta_{y}^{1 / 2} k_{2 s} d s \exp \left(i \psi_{y}\right)\right] \rightarrow 0 ; & \sum_{j}\left[\beta_{y}^{3 / 2} k_{2 s} d s \exp \left(i \psi_{y}\right)\right] \rightarrow 0 \\
\sum_{j}\left[\beta_{x} \beta_{y}^{1 / 2} k_{2 s} d s \exp \left(i\left(2 \psi_{x} \pm \psi_{y}\right)\right)\right] \rightarrow 0 ; & \sum_{j}\left[\beta_{y}^{3 / 2} k_{2 s} d s \exp \left(i 3 \psi_{y}\right)\right] \rightarrow 0
\end{array}
$$

where the summations are taken across the IR. The correction weight factors for these resonances are $\beta_{x}^{1 / 2} \beta_{y}$ and $\beta_{y}^{3 / 2}$. Under the assumptions of negligible betatron phase advances in the triplets and the $\pi$ phase advances between the triplets, the cancellation of the five resonance strengths generated by skew sextupoles, from (14), requires two conditions:

$$
\left\{\begin{array}{l}
\sum_{L} k_{2 s} \beta_{x} \beta_{y}^{1 / 2} d s-\sum_{R} k_{2 s} \beta_{x} \beta_{y}^{1 / 2} d s=0 \\
\sum_{L} k_{2 s} \beta_{y}^{3 / 2} d s-\sum_{R} k_{2 s} \beta_{y}^{3 / 2} d s=0
\end{array}\right.
$$

The tune shifts from skew sextupoles are produced by a vertical bump and are given by:

$$
\left\{\begin{array}{l}
\Delta Q_{x}=\frac{1}{4 \pi} \sum k_{2 s} \beta_{x} y_{c o} d s \propto\left(\sum_{L} k_{2 s} \beta_{x} \beta_{y}^{1 / 2} d s-\sum_{R} k_{2 s} \beta_{x} \beta_{y}^{1 / 2} d s\right) \frac{A_{\text {bump }}}{4 \pi \beta_{y c}^{1 / 2}} \\
\Delta Q_{y}=-\frac{1}{4 \pi} \sum k_{2 s} \beta_{y} y_{c o} d s \propto-\left(\sum_{L} k_{2 s} \beta_{y}^{3 / 2} d s-\sum_{R} k_{2 s} \beta_{y}^{3 / 2} d s\right) \frac{A_{b u m p}}{4 \pi \beta_{y c}^{1 / 2}}
\end{array}\right.
$$

where $\beta_{y c}$ is the vertical beta-function at the central corrector of the bump. Similarly to the sextupole correction, two IR skew sextupole correctors can eliminate the linear dependence of the horizontal and vertical tune shifts with respect to the vertical IR bump amplitude. Since the tune shift correction fulfills the conditions of (15), the skew sextupole resonance harmonics are also corrected by eliminating the tune shifts. 


\section{Octupole effects: resonances and tune shifts}

The perturbation Hamiltonian for an octupole error is given by

$$
\Delta H=\frac{k_{3}}{24}\left(x^{4}-6 x^{2} y^{2}+y^{4}\right)
$$

where the octupole strength $k_{3}$ is proportional to $b_{3}$ magnet error:

$$
k_{3}=\frac{1}{(B \rho)} \frac{\partial^{3} B_{y}}{\partial x^{3}}=\frac{6 B_{N} b_{3}}{(B \rho) R_{r}^{3}}
$$

Substituting (7) into (17) and expanding, it follows that octupoles generate the $4 Q_{x}=p$, $4 Q_{y}=p, 2 Q_{x}=p$, and $2 Q_{x} \pm 2 Q_{y}=p$ resonances. For resonance correction, it is necessary to minimize all the following resonance strengths:

$$
\begin{array}{ll}
\sum_{j}\left[\beta_{x}^{2} k_{3} d s \exp \left(i 4 \psi_{x}\right)\right]_{j} \rightarrow 0 ; & \sum_{j}\left[\beta_{y}^{2} k_{3} d s \exp \left(i 4 \psi_{y}\right)\right]_{j} \rightarrow 0 \\
\sum_{j}\left[\beta_{x}^{2} k_{3} d s \exp \left(i 2 \psi_{x}\right)\right]_{j} \rightarrow 0 ; & \sum_{j}\left[\beta_{y}^{2} k_{3} d s \exp \left(i 2 \psi_{y}\right)\right]_{j} \rightarrow 0 \\
\sum_{j}\left[\beta_{x} \beta_{y} k_{3} d s \exp \left(i 2 \psi_{x}\right)\right]_{j} \rightarrow 0 ; & \sum_{j}\left[\beta_{x} \beta_{y} k_{3} d s \exp \left(i 2 \psi_{y}\right)\right]_{j} \rightarrow 0 \\
\sum_{j}\left[\beta_{x} \beta_{y} k_{3} d s \exp \left(i\left(2 \psi_{x} \pm 2 \psi_{y}\right)\right)\right]_{j} \rightarrow 0 &
\end{array}
$$

Three different weight factors $\beta_{x}^{2}, \beta_{y}^{2}$ and $\beta_{x} \beta_{y}$ play a role for different resonances. For a round beam, the leading resonances in the horizontal and vertical planes are $4 Q_{x}=p$ and $4 Q_{y}=p$, with weight factors respectively $\beta_{x}^{2}$ and $\beta_{y}^{2}$. For an IR with $\pi$ phase advance between the triplets, the condition for resonance compensation leads to three conditions:

$$
\left\{\begin{array}{l}
\sum_{L} k_{3} \beta_{x}^{2} d s+\sum_{R} k_{3} \beta_{x}^{2} d s=0 \\
\sum_{L} k_{3} \beta_{x} \beta_{y} d s+\sum_{R} k_{3} \beta_{x} \beta_{y} d s=0 \\
\sum_{L} k_{3} \beta_{y}^{2} d s+\sum_{R} k_{3} \beta_{y}^{2} d s=0
\end{array}\right.
$$


If a horizontal plane IR bump is used, the tune shifts from the IR octupoles are proportional to the square of the bump amplitude:

$$
\left\{\begin{array}{l}
\Delta Q_{x}=\frac{1}{8 \pi} \sum k_{3} \beta_{x} x_{c o}^{2} d s \propto\left(\sum_{L} k_{3} \beta_{x}^{2} d s+\sum_{R} k_{3} \beta_{x}^{2} d s\right) \frac{A_{x b u m p}^{2}}{8 \pi \beta_{x c}} \\
\Delta Q_{y}=-\frac{1}{8 \pi} \sum k_{3} \beta_{y} x_{c o}^{2} d s \propto-\left(\sum_{L} k_{3} \beta_{x} \beta_{y} d s+\sum_{R} k_{3} \beta_{x} \beta_{y} d s\right) \frac{A_{x b u m p}^{2}}{8 \pi \beta_{x c}}
\end{array}\right.
$$

Unlike the cases previously considered, sextupole and skew-sextupole, a tune shift compensation using only a bump in one plane does not provide total compensation for octupole errors since not all of the conditions of (20) are satisfied. Therefore in addition to the horizontal bump one has to correct tune shift caused by a vertical orbit bump:

$$
\left\{\begin{array}{l}
\Delta Q_{x}=\frac{1}{8 \pi} \sum k_{3} \beta_{x} y_{c o}^{2} d s \propto\left(\sum_{L} k_{3} \beta_{x} \beta_{y} d s+\sum_{R} k_{3} \beta_{x} \beta_{y} d s\right) \frac{A_{y b u m p}^{2}}{8 \pi \beta_{y c}} \\
\Delta Q_{y}=-\frac{1}{8 \pi} \sum k_{3} \beta_{y} y_{c o}^{2} d s \propto-\left(\sum_{L} k_{3} \beta_{y}^{2} d s+\sum_{R} k_{3} \beta_{y}^{2} d s\right) \frac{A_{y b u m p}^{2}}{8 \pi \beta_{y c}}
\end{array}\right.
$$

A minimum of three octupole correctors are needed to make the full compensation of the tune shift and consequently of the resonance strengths.

\section{Skew octupole effects: resonances and tune shifts}

The perturbation Hamiltonian for a skew octupole error is given by

$$
\Delta H=\frac{k_{3 s}}{6}\left(x^{3} y-x y^{3}\right)
$$

where the skew octupole strength $k_{3 s}$ is proportional to the $a_{3}$ magnet error:

$$
k_{3 s}=\frac{1}{(B \rho)} \frac{\partial^{3} B_{x}}{\partial x^{3}}=\frac{6 B_{N} a_{3}}{(B \rho) R_{r}^{3}}
$$

Substituting (7) into (23) and expanding, one can see that skew octupoles can generate the $Q_{x} \pm Q_{y}=p, 3 Q_{x} \pm Q_{y}=p, Q_{x} \pm 3 Q_{y}=p$ resonances. For full correction, the following resonance strengths should be minimized: 


$$
\begin{aligned}
& \sum_{j}\left[\beta_{x}^{3 / 2} \beta_{y}^{1 / 2} k_{3 s} d s \exp \left(i\left(\psi_{x} \pm \psi_{y}\right)\right)\right]_{j} \rightarrow 0 ; \sum_{j}\left[\beta_{x}^{1 / 2} \beta_{y}^{3 / 2} k_{3 s} d s \exp \left(i\left(\psi_{x} \pm \psi_{y}\right)\right)\right]_{j} \rightarrow 0 \\
& \sum_{j}\left[\beta_{x}^{3 / 2} \beta_{y}^{1 / 2} k_{3 s} d s \exp \left(i\left(3 \psi_{x} \pm \psi_{y}\right)\right)\right]_{j} \rightarrow 0 ; \sum_{j}\left[\beta_{x}^{1 / 2} \beta_{y}^{3 / 2} k_{3 s} d s \exp \left(i\left(\psi_{x} \pm 3 \psi_{y}\right)\right)\right]_{j} \rightarrow 0
\end{aligned}
$$

where the sums are again taken across the IR. Under the usual assumption of negligible betatron phase advances in the triplets and the $\pi$ phase advances between the triplets, there are two different weight factors $\beta_{x}^{1 / 2} \beta_{y}^{3 / 2}$ and $\beta_{x}^{3 / 2} \beta_{y}^{1 / 2}$ and two resonance strength cancellation conditions:

$$
\left\{\begin{array}{l}
\sum_{L} k_{3 s} \beta_{x}^{3 / 2} \beta_{y}^{1 / 2} d s+\sum_{R} k_{3 s} \beta_{x}^{3 / 2} \beta_{y}^{1 / 2} d s=0 \\
\sum_{L} k_{3 s} \beta_{x}^{1 / 2} \beta_{y}^{3 / 2} d s+\sum_{R} k_{3 s} \beta_{x}^{1 / 2} \beta_{y}^{3 / 2} d s=0
\end{array}\right.
$$

According to Table 1 separate horizontal or vertical orbit bumps lead in this case to linear coupling excitation. Nevertheless, using simultaneously horizontal and vertical orbit bumps can create the tune shift due to gradient feed-down. Assuming equal bump amplitudes, $A_{x b u m p}=A_{y b u m p}=A_{\text {bump }}$, the tune shifts produced by this diagonal bump are:

$$
\left\{\begin{array}{l}
\Delta Q_{x}=\frac{1}{4 \pi} \sum k_{3 s} \beta_{x} x_{c o} y_{c o} d s \propto\left(\sum_{L} k_{3 s} \beta_{x}^{3 / 2} \beta_{y}^{1 / 2} d s+\sum_{R} k_{3 s} \beta_{x}^{3 / 2} \beta_{y}^{1 / 2} d s\right) \frac{A_{b u m p}^{2}}{4 \pi \beta_{x c}^{1 / 2} \beta_{y c}^{1 / 2}} \\
\Delta Q_{y}=-\frac{1}{4 \pi} \sum k_{3 s} \beta_{y} x_{c o} y_{c o} d s \propto-\left(\sum_{L} k_{3 s} \beta_{x}^{1 / 2} \beta_{y}^{3 / 2} d s+\sum_{R} k_{3 s} \beta_{x}^{1 / 2} \beta_{y}^{3 / 2} d s\right) \frac{A_{b u m p}^{2}}{4 \pi \beta_{x c}^{1 / 2} \beta_{y c}^{1 / 2}}
\end{array}\right.
$$

Therefore one can use a diagonal IR bump and two skew octupole correctors to cancel the quadratic in the bump amplitude terms of the horizontal and vertical tune shifts. According to (26), it also compensates the skew octupole resonance strengths.

In conclusion, with an appropriate choice of orbit bumps the compensation of tune shifts dependence on the bump amplitude is equivalent to the compensation of the corresponding nonlinear resonance strength. The correct choice of orbit bump is: a horizontal bump for the sextupole correction, a vertical bump for the skew sextupole correction, both horizontal and vertical bumps for the octupole correction and a diagonal bump for the skew octupole correction. 


\section{Beam-based IR correction: technique and results}

An operational environment has been developed at RHIC to implement local IR corrections with the IR bump technique. After a description of the RHIC IR regions, we will describe the correction operational tools, procedure and results. Sextupole IR correction is now operational and part of the machine set-up for the RHIC run. IR sextupole corrections have been validated by lifetime observations and dynamic aperture measurements. Octupole and higher order correction activity has started and we will discuss the potential and limits of this technique.

\section{The RHIC IR layout and correctors}

RHIC consists of 2 super-conducting rings, top energy of $100 \mathrm{GeV} / \mathrm{u}$, circumference of $\sim 3900 \mathrm{~m}$, designed to accelerate and collide a variety of species, from polarized protons to fully stripped gold ions. The lattice has approximate 6-fold symmetry: 6 FODO arcs matched to IRs with $\beta^{*}$ tunable from $10 \mathrm{~m}$ to $0.9 \mathrm{~m}$.

Figure 1: Top schematic view of one low $\beta^{*}$ RHIC Interaction Region (IR8)

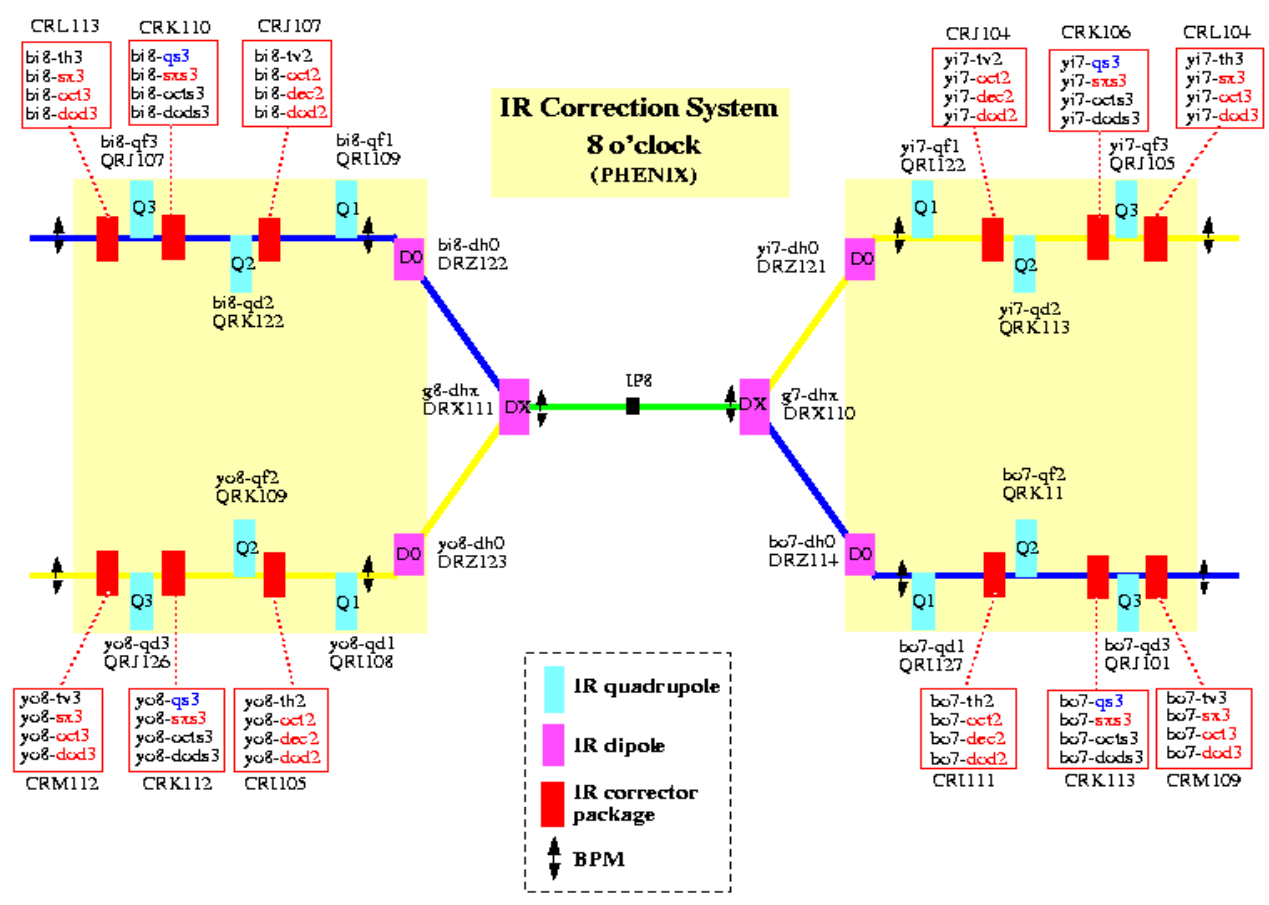


The typical lattice configuration for operations has the 6 IR 's tuned to $\beta^{*}=10 \mathrm{~m}$ at injection. $\beta^{*}$ is squeezed on the ramp to $5 \mathrm{~m}$ in all IRs at transition. The experimental IRs are squeezed further in the last part of the ramp to $1 \mathrm{~m}$ (or $0.9 \mathrm{~m}$ ) in IR6 and IR8 (hosting respectively the high-luminosity detectors STAR and Phenix) and to 3m in IR10 and IR2 (hosting the smaller detectors Phobos and Brahms). IR10 and IR4 are service IRs and are constant at $\beta^{*}=5 \mathrm{~m}$ after transition. Figure 1 is a schematic view of IR 8 .

The beams (blue and yellow) are horizontally separated after the interaction point (IP) by the DX and D0 magnets and focused by the triplets (Q1 Q2 and Q3). Every triplet has three 4-layers corrector packages, as specified in Table 2.

Table 2: RHIC IR correction package

\begin{tabular}{|l|l|l|l|}
\hline Corrector package & Corrector layer & Corrector symbol & Power supply \\
\hline C1 (normal) & Dipole & th, tv & yes \\
\hline & Octupole & oct2 & yes \\
\hline & Decapole & dec2 & yes \\
\hline & Dodecapole & dod2 & yes \\
\hline C2 (skew) & Skew quadrupole & qs3 & yes \\
\hline & Skew sextupole & sxs3 & yes \\
\hline & Skew octupole & octs3 & no \\
\hline & Skew dodecapole & dods3 & no \\
\hline C3 (normal) & Dipole & th, tv & yes \\
\hline & Sextupole & sx3 & yes \\
\hline & Octupole & oct3 & yes \\
\hline & Dodecapole & dod3 & yes \\
\hline
\end{tabular}

Correction packages containing horizontal and vertical dipole corrector magnets are placed at locations with respectively large horizontal and vertical $\beta$. In particular, the correction package includes 2 octupole and 2 dodecapole correctors for each triplet and 1 sextupole per triplet. Although the magnet correction packages are installed at every IR in the machine, presently only the correctors in IR6 and IR8 (tuned to $\beta^{*}=0.9-1 \mathrm{~m}$ in collision) are powered by bipolar $50 \mathrm{~A}$ corrector supplies. Correctors are also not yet connected to the skew octupole and dodecapole layers. 


\section{Linear corrections}

The skew quadrupole correctors in package $\mathrm{C} 2$ are routinely used to compensate the local coupling effect of the IR triplets arising from residual roll alignment errors. The skew quadrupole correction strengths have been operationally set by minimizing the vertical RMS orbit in the arcs generated by a $5-10 \mathrm{~mm}$ horizontal bump in each individual triplet. Measuring the horizontal orbit RMS in the arcs generated by a vertical bump in the triplets has then tested the quality of the corrections: this verified that the corrector effectively minimizes the RMS orbit in both planes. The success of the local linear IR correction opened up the possibility of non-linear local corrections.

As already discussed, it is possible in principle to infer local non-linear effects both from the measure of residual RMS orbit and of tune shifts generated by local orbit bump in the IR. Given existing limitations on the resolution of the orbit measurement and on the allowable bump amplitude at the triplets, in practice we used so far almost exclusively the measurement of tune shift as a function of bump amplitude for non-linear correction.

\section{Tune diagnostics}

High-resolution tune measurement is a key requirement for non-linear corrections. Of the tune measurement techniques available at RHIC, FFT of turn-by-turn BPM data, tune meter kicker, Schottky detector and Phase Lock Loop (PLL), only the latter offer the continuous tune signal and the resolution required to make the measurement practical. The PLL, a $245 \mathrm{MHz}$ system, in high-resolution mode gives tune data at $100 \mathrm{~Hz}$ and the resolution can be better than $5 \times 10^{-5}$. The PLL in lower resolution mode is used operationally for routine tune and chromaticity measurements on the ramp.

\section{IR bump application}

A control room application has been developed to set-up IR bumps and to collect and analyze data for IR corrections. The application provides communication to power supply and ramp managing software for IR bump set-up and activation, reads in and plots PLL and orbit data in real time and allows online data analysis to support operational corrections. A view of the graphic user interface (Figure 2) summarizes its functionality. 
Figure 2. Application interface to set-up, run and analyze data for IR corrections

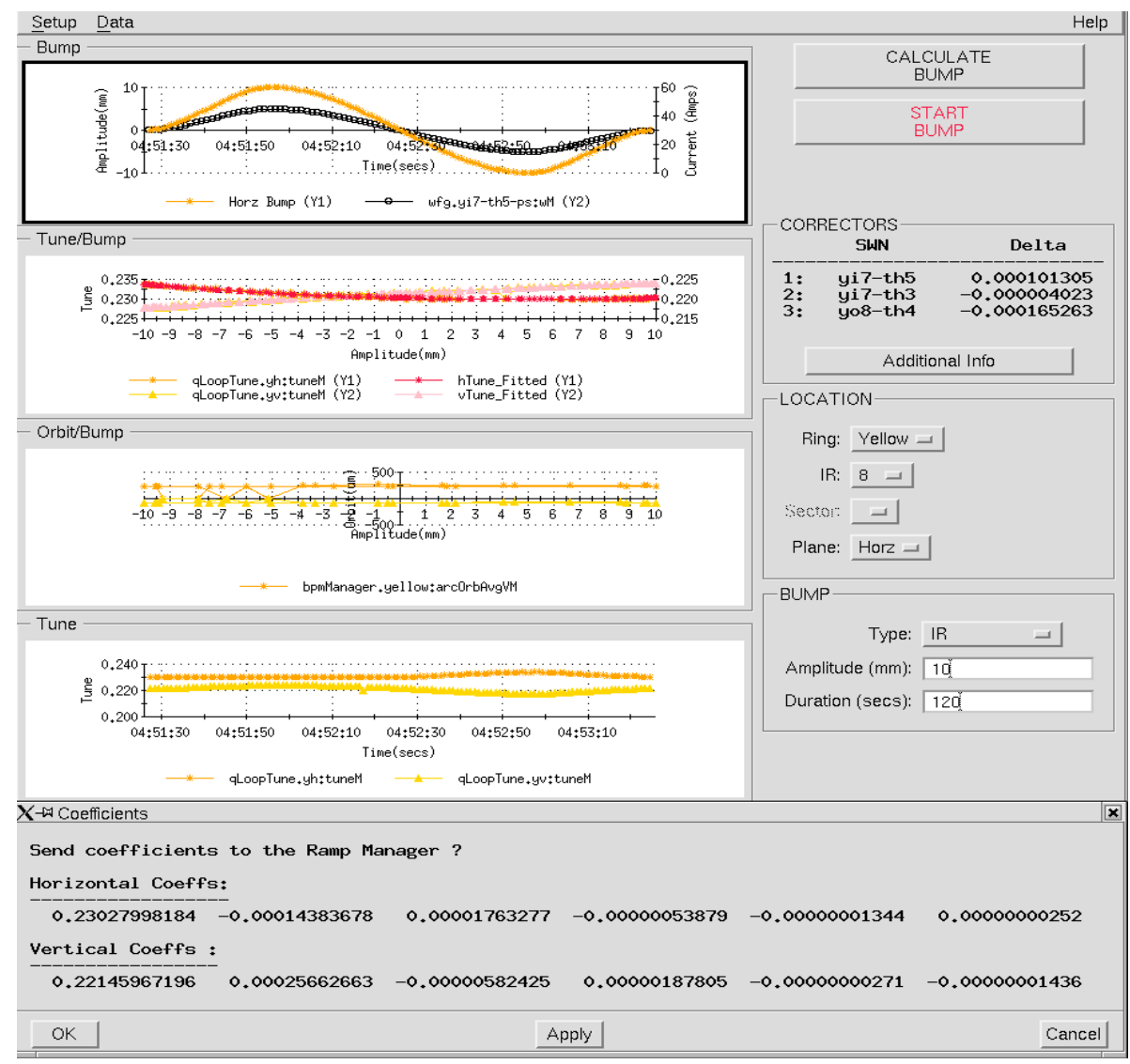

We can set-up several types of IR bumps for different purposes. A schematic view of bump set-up is shown in Figure 3. Triplet bumps, 3-bump centered at one triplet, are used for local correction at the individual triplet (possible for skew quadrupole, octupole, dodecapole). The bumps across the IR span 2 triplets: in this case we measure the integrated effect of the IR quadrupoles: as we will discuss, this is used for sextupole correction. It is worth noticing that in order to correctly measure errors from the IR magnet only, the dipole correctors must not be interleaved with the IR magnets, so for the bump across the IR we still use only 3 dipole correctors, using the natural property of the IR optics to produce a bump anti-symmetric with respect to the interaction point. The angle bump, a 4-bump across the IR centered at the IP is also used to gather beam-beam tune shift information as a function of crossing angle. The application allows to set up bumps at every IR, independently in the Blue and Yellow ring, and to define the maximum bump amplitude and the duration. During the typical bump duration of 60-120 
sec the orbit bump is ramped from zero to maximum amplitude, positive first, then negative. Tune $(100 \mathrm{~Hz})$ and RMS orbit data from the arcs $(0.25-1 \mathrm{~Hz})$ are collected and displayed as a function of time and as a function of bump amplitude. The application allows polynomial fitting of the tune shift and orbit data as a function of bump amplitude, which is useful to analyze online the multi-pole effects and to calculate the correction necessary to cancel the tune shifts order-by-order.

Figure 3. Schematic view of IR bump set-up

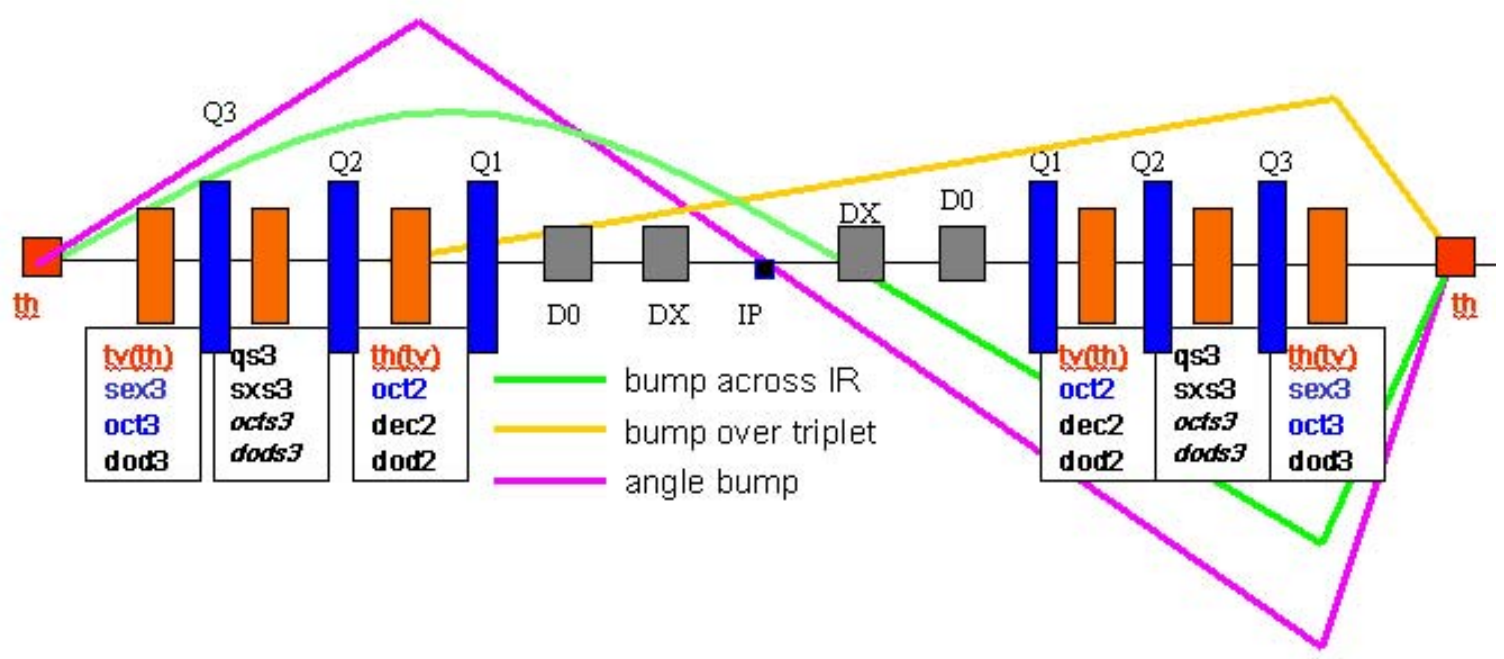

\section{Experimental set-up for IR measurements and correction}

Good operational preparation is needed for successful IR measurements and correction, in particular:

- The 2 rings must be anti-cogged, i.e. longitudinally separated, by at least 3 RF buckets to avoid beam-beam effects

- Measurements are performed at the beginning of a ramp, when the transverse emittance is smaller, giving larger amplitude available for the bumps. Typically we use dedicated ramps of 6 bunches (instead of the 45-56 typically used for operations) as this is enough for good PLL measurements but not enough overall beam intensity to risk dangerous quenches in case of accidental beam loss.

- Both rings must be well de-coupled, with coupling corrected to a minimum tune separation $\Delta \mathrm{Q}_{\min }<0.002$. 
- The horizontal and vertical fractional tunes are separated before the measurements by 0.01-0.012 to further minimize coupling effects. If the tunes get too close during the bump $(<0.002-0.003)$, that can interfere with the PLL lock in highresolution mode.

- Good overall orbit correction with horizontal and vertical orbit rms $<1 \mathrm{~mm}$

- Good centering of the orbit in the IR triplets, to insure symmetry during the measurement. Typically, local orbit adjustments in the IR regions are necessary prior to the measurement and correction to reach a residual orbit error of 1-2 mm in the IR magnets, a practical limit so far due to BPM alignment.

The choice of bump amplitude is a critical one. One the one hand, larger maximum bump amplitude is desirable because that enhances the measured effect - and that is particularly important for higher-order effects, as we will discuss. On the other hand there are practical aperture considerations to avoid beam losses in the triplets during the bumps (potentially causing beam aborts) and practical limits on the available strengths of the IR dipole correctors, as the strength for the IR bump adds to the strength of the pre-existing orbit corrections. Maximum bump amplitudes of $+/-5 \mathrm{~mm}$ proved enough to resolve sextupole effects, of $+/-10 \mathrm{~mm}$ for octupole effects, and $+/-15 \mathrm{~mm}$ for higher orders.

\section{Figure 4. Optimization of horizontal orbit in IR8, yellow ring, prior to IR correction}
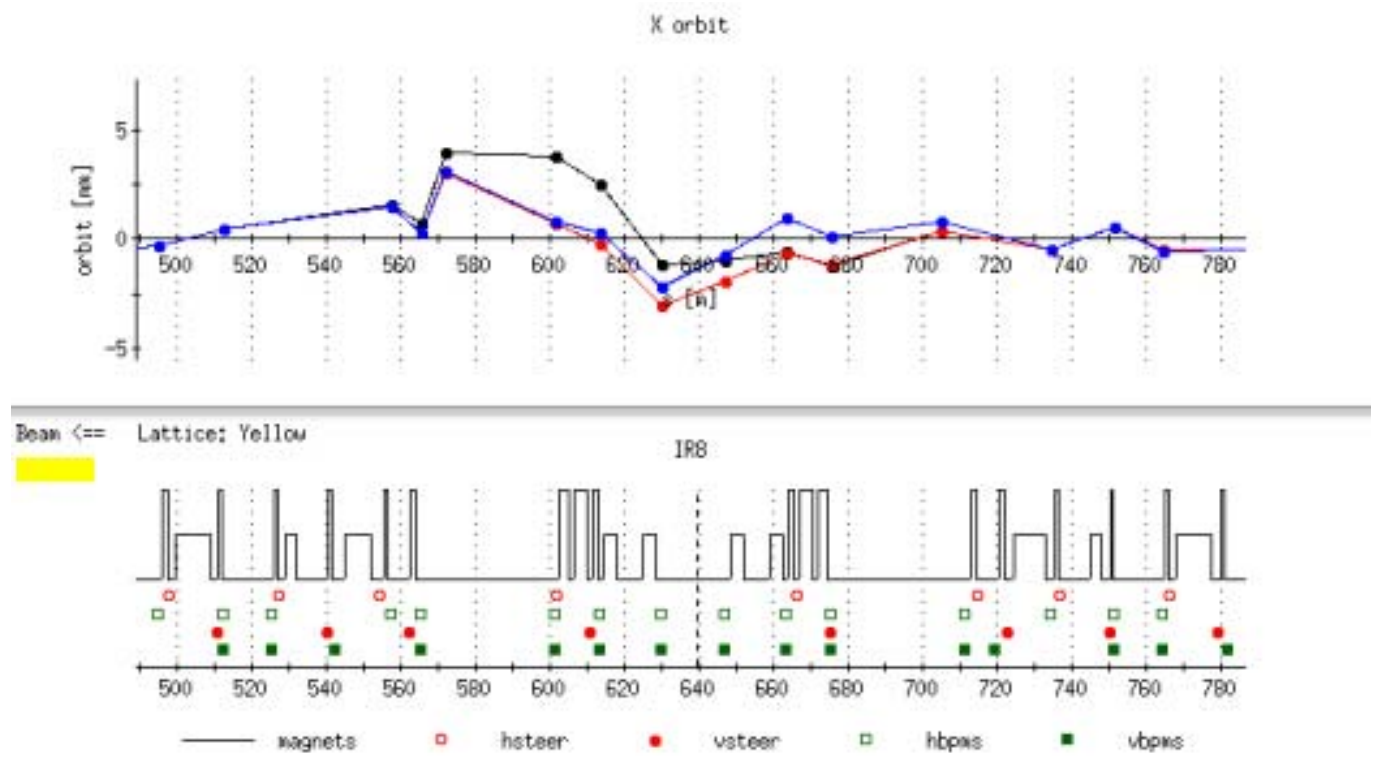


\section{IR sextupole effects and correction}

The feasibility of local IR sextupole correction has been tested and verified during RHIC Run-1 (Au and P-P collisions); the IR bumps dedicated application was developed for Run-3 (d-Au and P-P collisions) and the procedure became operational for Run-4 (Au and P-P collisions) and Run-5.

The sextupole correction proved to work for all machine and optics configuration we have run so far at RHIC. Table 3 summarizes the IR sextupole correctors strengths necessary to operationally cancel the linear dependence of tune shift versus bump amplitude, that typically is in the range of $0.00003-0.0003 / \mathrm{mm}$ for different IRs and different machine configurations.

Table 3. Sextupole corrector strengths to cancel the linear dependence (sextupole term) of the tune shift vs. IR bump amplitude

\begin{tabular}{|l|l|l|l|l|l|}
\hline $\begin{array}{l}\text { IR sextupole } \\
\text { corrector }\end{array}$ & $\begin{array}{l}\text { Run-3 d-Au } \\
\beta^{*}=2 \mathrm{~m} \\
\mathrm{I}(\mathrm{Q})=4500 \mathrm{~A}\end{array}$ & $\begin{array}{l}\text { Run-3 P-P } \\
\beta^{*}=1 \mathrm{~m} \\
\mathrm{I}(\mathrm{Q})=2000 \mathrm{~A}\end{array}$ & $\begin{array}{l}\text { Run-4 Au } \\
\beta^{*}=1 \mathrm{~m} \\
\mathrm{I}(\mathrm{Q})=4500 \mathrm{~A}\end{array}$ & $\begin{array}{l}\text { Run-4 P-P } \\
\beta^{*}=1 \mathrm{~m}(\mathrm{Q})=2000 \mathrm{~A}\end{array}$ & $\begin{array}{l}\text { Run-5 Cu } \\
\beta^{*}=85 \mathrm{~m} \\
\mathrm{I}(\mathrm{Q})=4500 \mathrm{~A}\end{array}$ \\
\hline Yo5-sx3 & -0.0014 & -0.003 & -0.006 & -0.001 & -0.007 \\
\hline Yi6-sx3 & +0.004 & 0.0 & +0.003 & +0.001 & +0.0035 \\
\hline Yi7-sx3 & +0.003 & +0.007 & +0.0005 & +0.001 & +0.003 \\
\hline Yo8-sx3 & -0.01 & -0.038 & 0.0 & -0.0012 & -0.003 \\
\hline Bi5-sx3 & +0.0012 & +0.001 & +0.0011 & -0.0022 & +0.0025 \\
\hline Bo6-sx3 & -0.004 & -0.003 & -0.001 & +0.001 & -0.005 \\
\hline Bo7-sx3 & 0.0 & -0.003 & 0.0 & -0.007 & -0.005 \\
\hline Bi8-sx3 & 0.0 & -0.0005 & 0.0 & +0.002 & +0.0025 \\
\hline
\end{tabular}

Table 3 compares the strengths during for different runs and IR optics configurations. There are 2 sextupole correctors per IR (Figure 1), one at a location of higher $\beta_{x}$

( $\beta_{x}=1278 \mathrm{~m}, \beta_{y}=480 \mathrm{~m}$ for the $\beta^{*}=1 \mathrm{~m}$ optics $)$ that is more effective in the horizontal plane, the other at a location of higher $\beta_{y}\left(\beta_{x}=471 \mathrm{~m}, \beta_{y}=1302 \mathrm{~m}\right)$, more effective in the 
vertical plane. The minimization of the sextupole needs to be done with the bump spanning the entire IR, and that includes potential effects from all IR triplets and the separator dipoles DX and D0. Although a bump of $5 \mathrm{~mm}$ is enough to measure the linear effect (sextupole), we typically use $10 \mathrm{~mm}$ bump amplitude to check the residual octupole and higher order effects.

The gold-gold ( $\mathrm{Au}-\mathrm{Au})$ and deuteron-gold data $(\mathrm{d}-\mathrm{Au})$ are taken at the collision energy of $100 \mathrm{GeV} / \mathrm{u}$ - with the main quadrupole bus powered at $\sim 4500 \mathrm{~A}$ - while the polarized proton (P-P) data are at $100 \mathrm{GeV}$, a lower energy, with the main quadrupole bus at 2000 A. As there is current (ramp) dependence of the magnetic errors in the IR dipole and quadrupoles, the errors configurations are different. Also, the quality of experimental setup, and the quality of correction improved over the runs (centering of orbit and coupling control). That can partially explain the different effects measured and corrected in P-P Run 3 and 4. Figure 5 shows an example of tune shift dependence on bump amplitude before (left) and after (right) local sextupole correction.

Figure 5. Tune shift versus bump amplitude in IR8, Yellow ring, before and after local sextupole correction

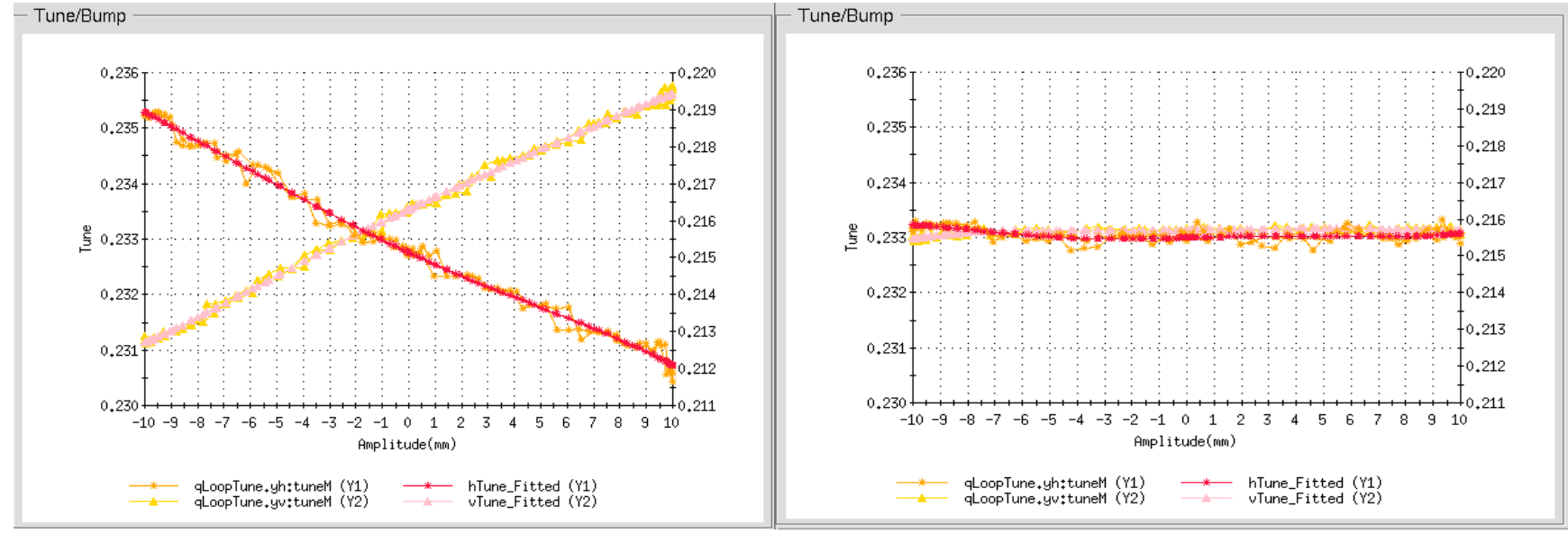

Figure 5 is an example of measurement and correction of an almost purely sextupole effect in IR8, Yellow Ring, in both planes. 


\section{IR skew sextupole effects and correction}

As discussed, a vertical bump provides all necessary information for the correction of skew sextupole effects. That was tested in IR8 in the yellow ring where we measured a substantial tune shift vs. vertical bump amplitude. Tune shifts before and after corrections are compared in Figure 6:

Figure 6. Tune shift from skew sextupole before and after correction
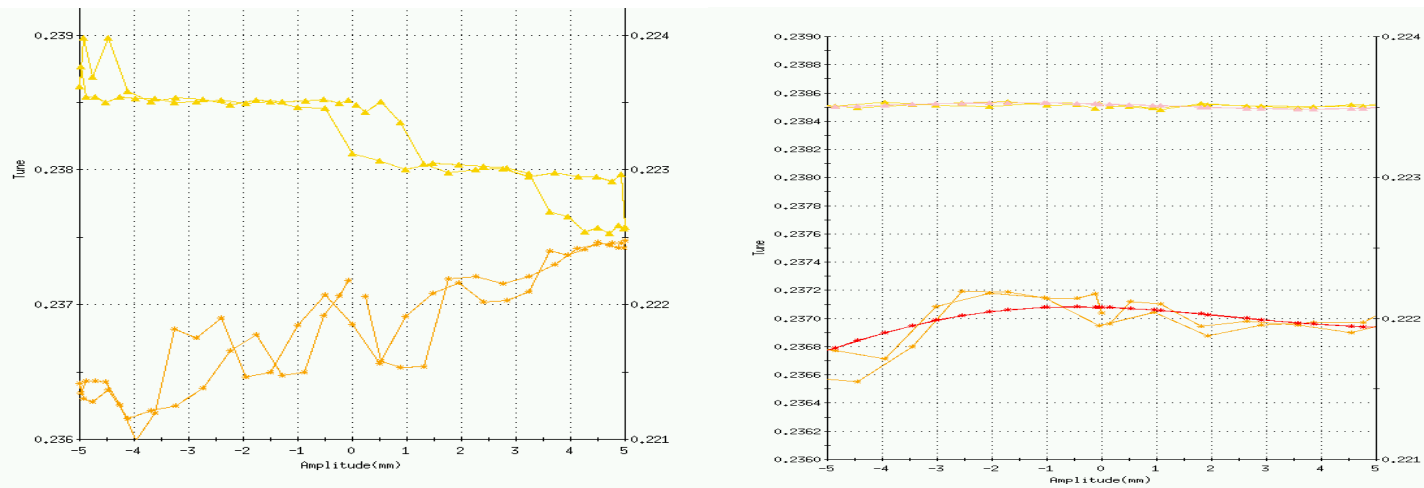

\section{IR octupole effects and correction test}

In order to help in the evaluation (and potential correction) of orders higher than sextupole we added the capability of online fitting and plotting the polynomial coefficients. We have 2 octupoles corrector layers per triplet (recall Figure 1), so it is in principle possible to correct for the octupole effect - i.e. the quadratic term in the fit tune shift versus bump amplitude, at every individual triplet.

Figure 7. Tunes shift from octupole before and after correction.
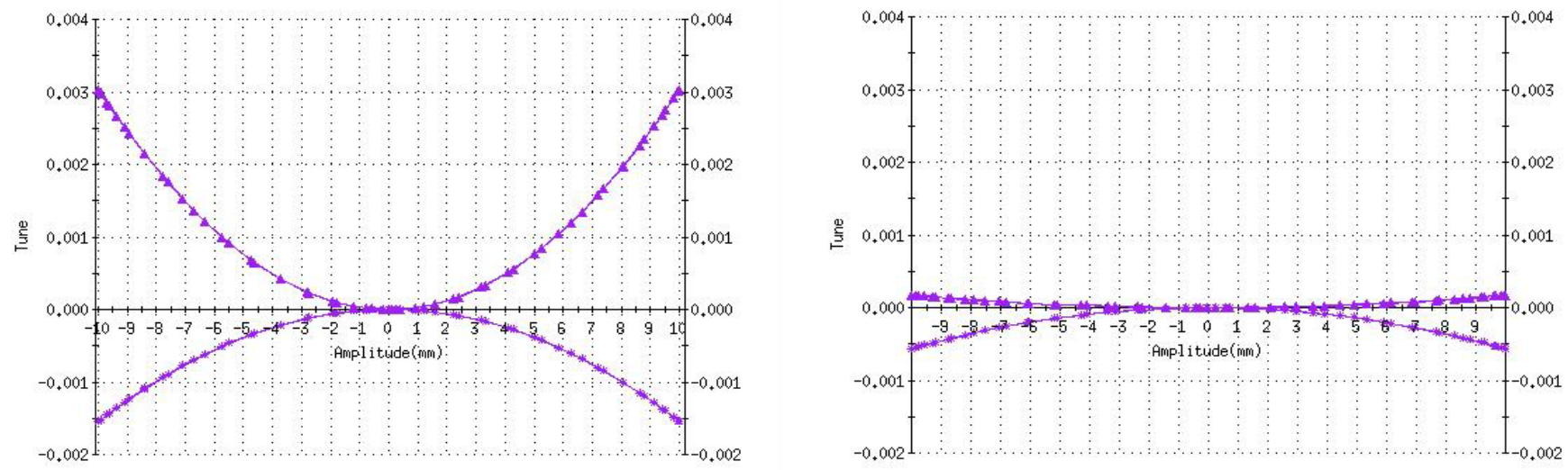
Figure 7 shows the result of correction of the quadratic dependence of the tune shift at one individual triplet with the 2 local octupole correctors. Though successful in minimizing the octupole tune shift, the octupole correction at the individual triplets was not used in operations. Since it required rather strong octupoles, the correction at the individual triplets affected negatively beam lifetime and measured dynamic aperture. In operation we minimized in Yellow IR8 the octupole quadratic dependence across the entire IR, similarly to what has been done for the sextupole correction. In this case the octupole strengths are small (see Table 3), and we have measured no adverse effect on lifetime and dynamic aperture.

\section{Higher order effects}

On-line fitting of polynomial coefficients allows us to evaluate the feasibility of measuring effects higher than octupole. For example, Figure 8 shows the polynomial fitting up to $4^{\text {th }}$ order for the dependence of tune versus bump amplitude, for a $15 \mathrm{~mm}$ horizontal and vertical bump centered in the IR8, yellow ring, during Run-5 $(\mathrm{Cu}-\mathrm{Cu}$ collisions).

Figure 8. Measurement of higher order in IR8, yellow ring.
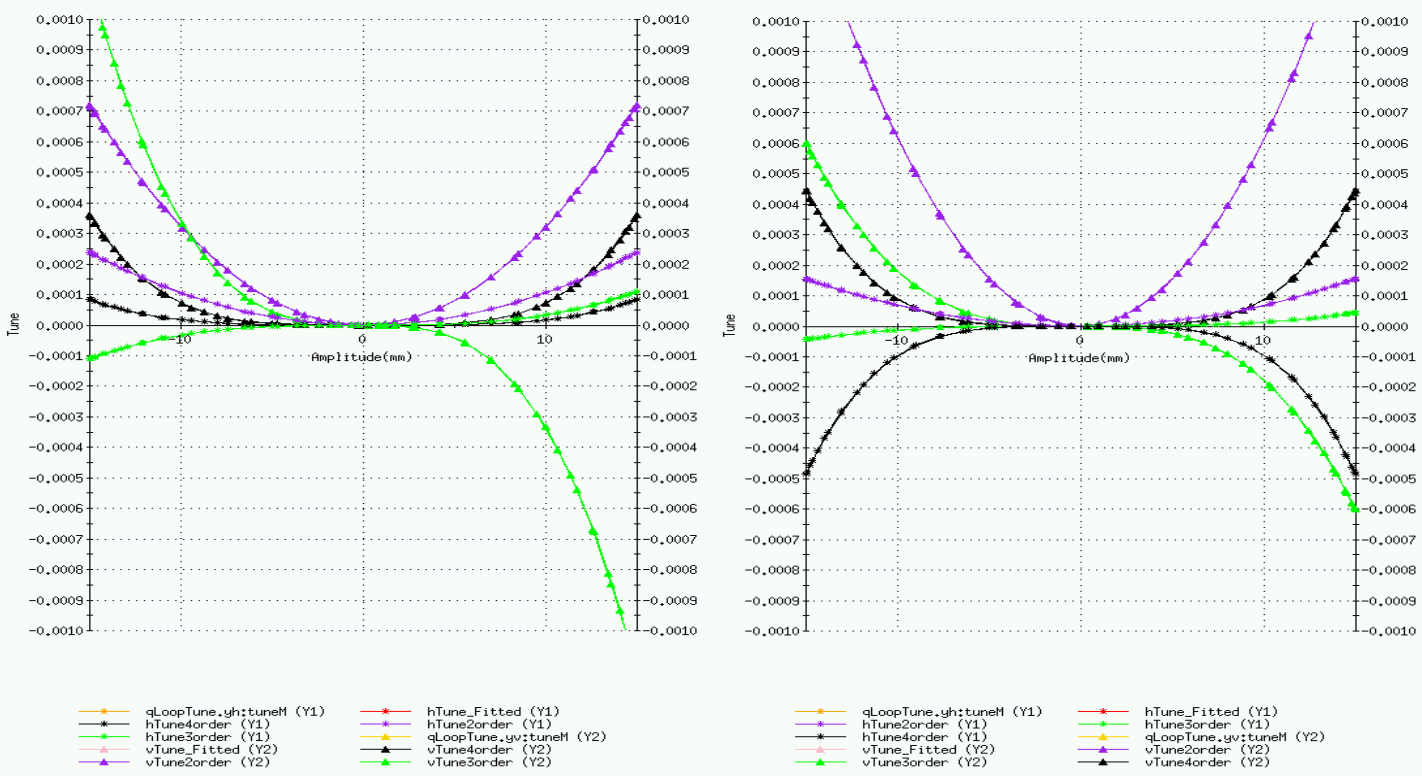
The left plot in Figure 8 shows the residual coefficients for the horizontal tune, (purple: octupole, green: decapole, black: dodecapole) after correction of the sextupole (linear) term. The plot on the right side of Figure 8 shows the change in the higher order harmonics after we powered up the yi7-dod3 dodecapole in IR8. A bump of $15 \mathrm{~mm}$ was necessary to resolve the effect, and that requires careful preparation of the beam (6 bunches of low emittance, good initial orbit). This is a practical limit at top energy, to go beyond $15 \mathrm{~mm}$ pencil beam could be used.

\section{IR correction model and magnetic measurements}

An effort is on going to compare the experimental non-linear effects measured in the IR with our present knowledge of the magnet data for the the IR triplets and separation dipole DX and D0. Accurate information about IR non-linear magnet fields is important for understanding of dynamic aperture, beam-beam limits, etc. The IR bump measurements have been used to benchmarking the measured magnet data. Figure 9 is a schematic view of the RHIC offline model.

\section{Figure 9: RHIC off-line simulation facility}

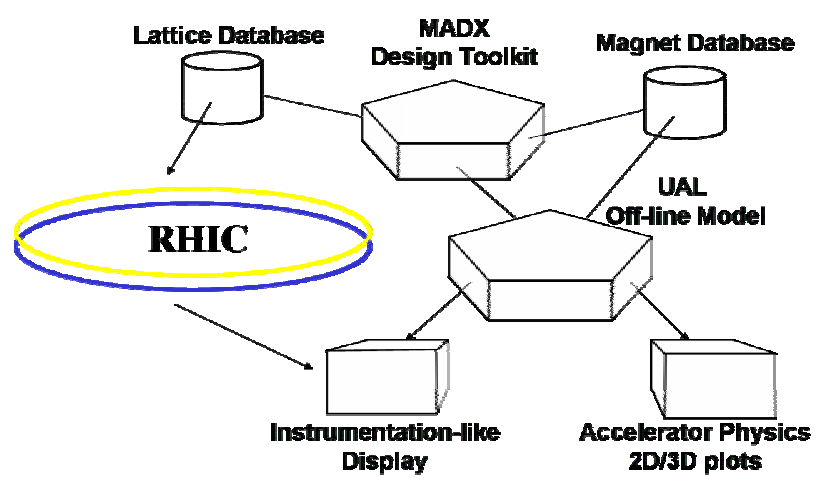

The model consists of three major components: MAD-X, UAL and ROOT. The MAD-X toolkit deals with all issues associated to lattice optics an design. The simulation applications proper are based on the UAL framework and open catalog of tracking algorithms. Finally, the ROOT toolkit facilitates the visualization of simulation results. Like the real accelerator, the off-line model is initialized from the design database, which 
ensures correspondence between linear optics in on-line machine operations and off-line studies. Moreover, to minimize possible discrepancy between operational and modeling scenarios, a part of the online IR bump application program was directly integrated into the off-line simulation environment.

The IR bump data measure the integrated tune shift induced by all magnets of the selected IR section. Every RHIC IR contains 6 triplet quadrupoles and 4 separation dipoles per ring. (See Figure 1). Every IR magnet either has been measured cold or warm, and in the latter case cold data are inferred from warm-to-cold correlations. Magnet field data for an IR magnet consist of about 60 parameters (including fringe fields and body harmonics) for each measured current. To efficiently analyze such a substantial amount of data, we have developed and employed a tune shift budget application based on the RHIC off-line simulation facility. The application reads the RHIC lattice including all

\section{Figure 10: Tune shift budget plots:}

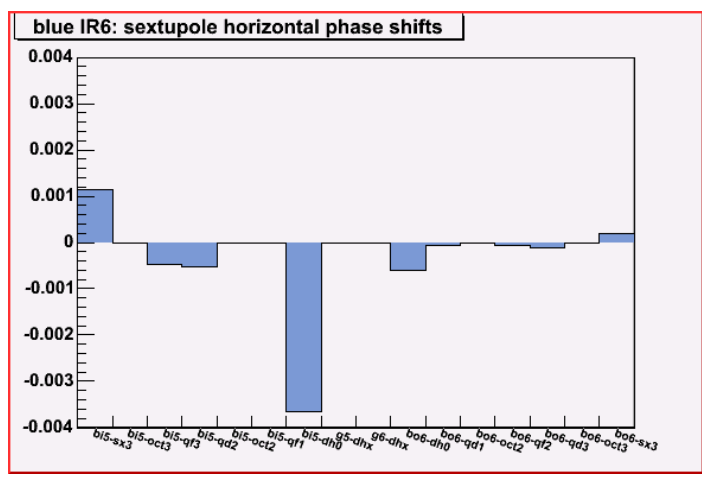

\section{a) Blue IR6}

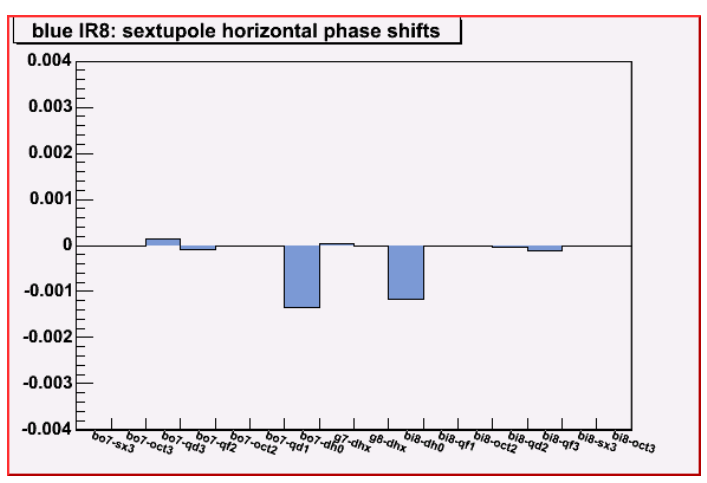

c) Blue IR8

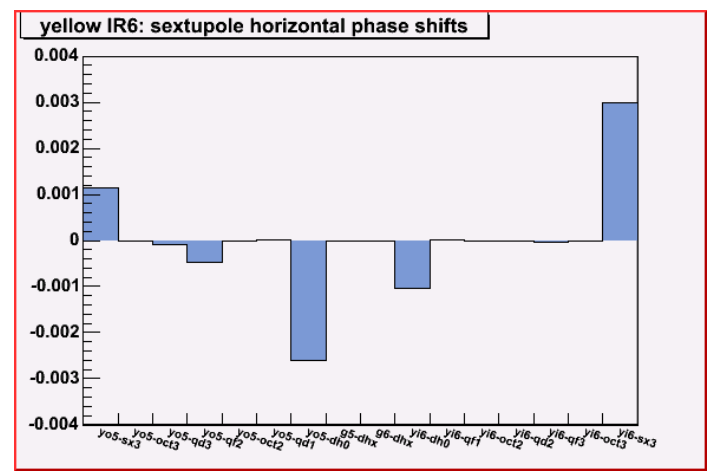

\section{b) Yellow IR6}

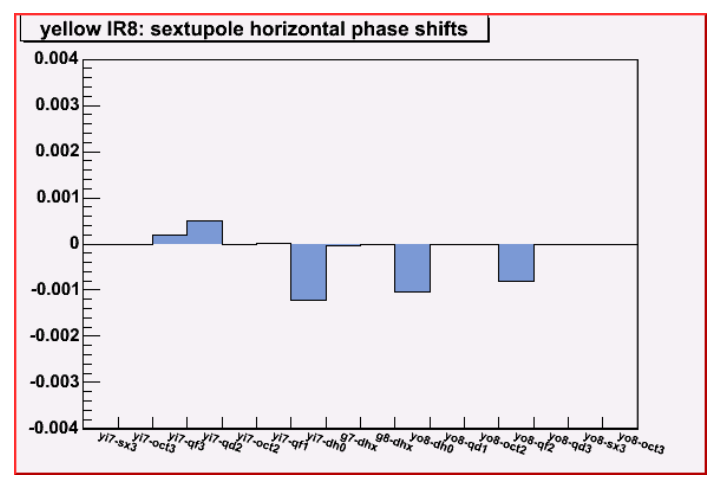

d) Yellow IR8 
non-linear magnet fields and non-linear operational correctors, creates IR bumps, and generates plots (See Figure 10) showing a set of individual element's tune shifts calculated according to equation 2. As described above, the online IR bump application is very flexible and enables to distinguish sextupole-driven linear tune shifts from the residual octupole and higher order effects. Then the off-line model has been initially applied to analysis of dominant sextupole harmonics. The plots of Figure 7 explicitly and generally identify the DH0 separation dipoles as the major source of sextupole error in the IRs. The comparison between the operationally measured sextupole tune shift and that predicted by the magnet measurements is summarized in Table 4 for different IRs.

Table 4. IR sextupole tune shift measured vs. predicted

\begin{tabular}{|l|l|l|}
\hline IR section & Measurement by IR bump & Off-line Model \\
\hline Blue IR6 & $-1.110^{-3}$ & $-4.210^{-3}$ \\
\hline Blue IR8 & $-2.010^{-3}$ & $-2.410^{-3}$ \\
\hline Yellow IR6 & $-3.910^{-3}$ & $-3.610^{-3}$ \\
\hline Yellow IR8 & $-0.510^{-3}$ & $-2.210^{-3}$ \\
\hline
\end{tabular}

The agreement in 2 cases is good. Analysis is in progress to understand the origin of discrepancy in the two other interaction regions.

\section{Conclusions}

The IR bump method allows an order-by-order compensation of tune shifts. It is equivalent to the compensation of corresponding non-linear resonance strengths, and more operational than the action-kick minimization [4]. The sextupole, skew-sextupole and octupole correction have been experimentally demonstrated and the sextupole 
correction is part of the RHIC machine operational set-up. Work on the measurement and correction of orders higher than octupole is in progress.

\section{References}

[1] F.Pilat, P. Cameron, V.Ptitsyn, BNL, J-P. Koutchouk, CERN, "Linear and Nonlinear Corrections in the RHIC IRs", EPAC2003

[2] P. Cameron, et al, PAC2003, ROAB009

[3] F.Pilat, S. Binello, P. Cameron, V. Ptitsyn, PAC2003, TPPB038

[4] Y. Luo et al., PAC 2005, MPPE030

\section{Acknowledgements}

The authors would like to thank Jean-Pierre Koutchouk, CERN, for the original formulation of the IR bump method and collaboration in the early phase of experimental work, P. Cameron, BNL, for continuous support and development of the essential PLL measurements, Sev Binello, BNL, for developing the IRbump application, and Jie Wei, BNL, for useful discussions. 